\title{
Sachverzeichnis
}

Aargau 192

Abberufung von Abgeordneten 70

Abstimmungsbeteiligung 25 ff., 79 f., $288 \mathrm{ff}$.

Abstimmungskampf $297 \mathrm{ff}$.

Agendainitiative $210 \mathrm{ff}$.

Alaska 166

Amtseintragung 263

Antragsquorum 79 ff., 94 ff., 138, 146, 218, $221 \mathrm{f} ., 259,262 \mathrm{f}$.

Anwendung der Direktdemokratie auf sich selbst $50 \mathrm{ff}$.

Appenzell 190, 192

Atomausstieg $198 \mathrm{f}$.

Ausgabenbremse 85

Ausländerstimmrecht 191

Ausschaffungsinitiative $176 \mathrm{f}$.

Ausschlußtatbestände 33, 48 ff., 84 ff., 114; siehe auch Finanztabu

Autonomiestatut für Südtirol 22, 31 ff.

Baden-Württemberg 323 f., 350 ff.

Basel-Landschaft 192

Basel-Stadt 191

Bayern 37, 44, 51, 53, 60, 111, 114, 135, 141, 155 f., 255 ff., 295 ff.

Beratung der Organisatoren 143, 219, 222

Berlin 51, 158, $321 \mathrm{ff}$.

Berliner Wassertisch $321 \mathrm{f}$.

Bern 20, 190 ff., 197 ff.

Beschleunigungswirkung direkter Demokratie $104 \mathrm{f}$.

Beteiligungsquorum 24 ff., 30 f., 80 f., 92, 95, 136, 140

Bindungswirkung direktdemokratischer Entscheidungen 84, 227 ff., $323 \mathrm{f}$.

Blockadepotential direkter Demokratie 46, 58, 117 f., 131, 133

Bozen 21 ff., 30

Brandenburg 114
Bremen 51, $135 \mathrm{ff}$.

„Bremse“ 43, 92 f.

Bundespräsident 61

Bundesrat 53 f., 61, 83, 108, 172

Bündnis 90/Die Grünen 116, 123 f., 141, 282, 292, 297, 310

Bürgerantrag 136, 142

Bürgerbegehren $328 \mathrm{ff}$.

Bürgerentscheid 21 f., 323 ff.

CDU 71, 116 f., 122, 133

CSU 60, 116 f., 122, 133, 276 f., 281, 287, $308,314,319$

Dezentralisierung $227 \mathrm{f}$.

Die Linke $116 \mathrm{f}$.

Direkte Demokratie auf Bundesebene 52 ff., 107 ff., 115 f., 292 f.

Direkte Demokratie auf kommunaler Ebene 56 f., 197 ff., 224 ff.

Direktwahl von Amtsträgern $101 \mathrm{f}$.

DVU 136

\section{EGMR 108}

Einfluß von Interessengruppen 125 f., 132

Eintragungs- bzw. Sammlungsfrist 114, 146

Einzelfallgesetz 377

Empirische Daten/Einstellung zur direkten Demokratie 111 f., 115 ff., 120 ff.

- Wirkung von Abstimmungskämpfen $296 \mathrm{ff}$.

\section{EMRK 34}

Entscheidungsfähigkeit der Bürger $86 \mathrm{f}$.

Entschleunigungswirkung direkter Demokratie 103

Erstentscheidungspflicht der Repräsentanten 78

Ethnisch-kulturelle Sensibilität/Identität $30 \mathrm{ff}$. 
Europäische Bürgerinitiative $201 \mathrm{ff}$.

Europäische Charta der Grundrechte 206

Europäische Union 95, 107 f., 111, 116, 201 ff., 244, 246, 253

Europäisches Parlament 204, 221

Europarat $203 \mathrm{f}$.

Ewigkeitsgebot $53 \mathrm{f}$.

FDP 116, 281 f., 287, 309, 311, 314, 319

Finanzierung der direkter Demokraite 104, $220,222,284 \mathrm{f}$.

Finanzierungsverantwortung $358 \mathrm{ff}$.

Finanzierungsverträge $352 \mathrm{ff}$.

Finanzkrise 237, $242 \mathrm{ff}$.

Finanztabu 49, 85, 110, 138 ff., 143 ff., 149, 154, 160 f., 233 f., 258 ff., 375, $384 \mathrm{ff}$.

Föderalismus, Föderalismusmodelle 54, 83

Forschung am Menschen 177

Frankreich 209, $224 \mathrm{ff}$.

Freie Stimmsammlung 146

Fribourg 18, 32, 192, 194

Fünfprozentklausel 80, 140, 149 f., 153

Funktionslogik $42 \mathrm{ff}$.

„Gaspedal“ 43 f., 92 f.

Genf $193 \mathrm{ff}$.

Gewaltenfusion $46 \mathrm{f}$., $58 \mathrm{f}$.

Gewaltenteilung 46, 66

GEWOBA 139, 145

Glarus 190

Gleichgeschlechtliche Ehe 13, 161 f., 165

Glücksspiel $163 \mathrm{f}$.

Graubünden 19, 190, 192

Griechenland 217

Großbritannien 59, 224, 247 ff.

Große Koalition 61

Großprojekte $87 \mathrm{ff}$.

Grundgesetz 48, 52 ff.
Hamburg 37, 44, 45, 49, 51, 56, 92, 140 , $155,157,324 \mathrm{ff}$.

Hessen 111, 114, 135, 141

Icesave $247 \mathrm{ff}$.

Imperatives Mandat $74 \mathrm{f}$.

Informiertheit der Abstimmenden 295 ff., $303 \mathrm{ff} ., 317 \mathrm{f}$.

Input-Legitimation $39 \mathrm{f}$.

Interesse am Volksentscheid 302 ff., 317

Island $237 \mathrm{ff}$.

Italien 14, 45, 60 f., 217

Jura 34, 191

Kalifornien 37, 102, 167

Kampagnenkontakte $299 \mathrm{ff}$.

Kampfhunde 196 f.

Kantonale Demokratie 15 ff., 186 ff.

Kompetenzordnung 355 ff., 376 f.

Konkordanzmodell 56

Konsensdemokratie 60

Kontralegislatur 149 f., 153

Kultur des Plebiszitären 89

Ladenöffnungszeiten 194

Landsgemeinde 190

Landtagswahlrecht $149 \mathrm{ff}$.

Lateinamerika 46

Legitimation durch Verfahren 76

Lerndruck $70 \mathrm{f}$.

Lissabon-Urteil 107, 109, 293

Litauen 45

Lobbyisten 39

Luzern 192

Maine 160 ff., 164, 166, 167 f.

Massachusetts 166

Materielle Konfliktlage 380 ff.

Mecklenburg 114

Mehr Demokratie e.V. 12, 50, 206 ff., 256 
Mehrwert direkter Demokratie 41 f., 70 f., 89, $102 \mathrm{ff}$.

Michigan 166

Minarettverbot 11, 13, 34, 37, 108 f., 169, $171 \mathrm{ff}$.

Minderheitenschutz 11 ff., 86, 110, $161 \mathrm{f}$.

Mitwirkung der Länder an der Bundesgesetzgebung $53 \mathrm{ff}$.

Nationalsozialismus 47

Neuenburg 191, 194

New Jersey 167

New Mexiko 163, 166

New York 167

Nichtraucherschutz 155 f., 181, 183, 193, 255 ff., 274 ff., 295 ff.

Nidwalden 192

Niederlande 209, 247 ff.

Nordrhein-Westfalen 114

North Dakota 166

Obwalden 192, 195

ÖDP 255, 277 ff., 297, 309, 311

Ohio 160, 163, $167 \mathrm{f}$.

Oneseat-Initiative $212 \mathrm{f}$.

Opposition 43, 46 f., 56, 58, 60 f., 94, 103

Organtreue 153

Österreich 14

Output-Legitimation $39 \mathrm{f}$.

Parlamentarische Behandlung des Volksbegehrens $146 \mathrm{ff}$.

Parlamentarische Demokratie 38 ff., 45 ff.

Parlamentarisches Budgetrecht 85

Parlamentarisches Regierungssystem 58 ff., 65 ff., $381 \mathrm{f}$.

Parlamentarisches System 58 ff.

Parlamentsauflösung durch Volksentscheid 93 f., 96, 136 f., 257

Parteien, Parteiendemokratie 38, 42, 55, 61 f., 67 f., 103, 114 ff.

Pfannenrevolution 242, 244
Plebiszit (i.e.S.) 114

Plebiszitäre Elemente $63 \mathrm{f}$.

Plebiszitäre Instrumente $63 \mathrm{f}$.

Plebiszitärer Cäsarismus 99

Pluralismus $70 \mathrm{f}$.

Politikverdrossenheit 117 f., 124, 132 f., 293

Populismus 89

Präsidentialismus 45 f., 56 f., 65, 225

Prinzipal/Agent $72 \mathrm{f}$.

Probit-Modell 119 f., 127 ff.

Quorum 49, 79 ff., 114; siehe Antrags-, Beteiligungs- und Zustimmungsquorum

Rationalität direkter Demokratie 38 ff., $86 \mathrm{ff}$.

Reaktiver Charakter direkter Demokratie $43 \mathrm{ff}$.

Rechtschreibreform 139

Referendum/bestätigendes 30, 32

- Bürgermeisterreferendum $230 \mathrm{ff}$.

- einfaches 58

- fakultatives 17, 43, 92 ff., 113, 160, 163, 168

- Icesave-Referendum $247 \mathrm{ff}$.

- in der Hand von Repräsentanten 75 f., $98 \mathrm{ff} ., 379 \mathrm{ff}$.

- konsultatives 100 f., 114, 225 ff.

- lokales $225 \mathrm{ff}$.

- nach Ablehnung einer Regierungsvorlage $379 \mathrm{ff}$.

- obligatorisches 17, 20, 58, 94 ff., 107 f., 113 f., 178, 268

- Verfassungsreferendum 135, 141, 268

Referendumsindustrie $103 \mathrm{f}$.

Reformpotential der direkten Demokratie $131 \mathrm{f}$.

Regel-Ausnahme-Verhältnis $42 \mathrm{f}$.

Regressionsvariablen $118 \mathrm{ff}$.

Rekrutierungsmuster 72 
Repräsentation und Demokratie 38 ff., $65 \mathrm{ff}$.

Repräsentationskonstitutives Konfliktpotential 74, 76

Repräsentative Demokratie $63 \mathrm{ff}$.

Reservefunktion direkter Demokratie 59

Responsivität 41, 72 f., 76, 78, 80, 90 f., 94, 96, 100

Rheinland-Pfalz 92

Rolling-Cross-Section-Design 298

Rückwirkungsverbot 378

Saarland 114

Sachlichkeitsgebot 324, $326 \mathrm{f}$.

Sachsen 114, 348 f.

Schaffhausen $192 \mathrm{f}$.

Schleswig-Holstein 155

Schweden 201, 217

Schweiz 92

Schweiz 11 ff., 30 ff., 37, 40, 43 f., 46 f., 50, 53 f., 79, 83, 87, 89, 92, 102, 108 ff., $114,122,169 \mathrm{ff}$.

Schwyz 192, 194

Selektionsdruck 78

Siegesdenkmal $21 \mathrm{f}$.

Slowakei 45

Slowenien 45

Solothurn 192 f.

Sozialdemokratie 47

Spanien 209, 217

SPD 71, 117, 122, 141, 297, 309 f., 354 f.

Sperrwirkung $323 \mathrm{f}$.

Sprachgruppen $15 \mathrm{ff}$.

St. Gallen 192 f., 198 f.

Ständemehr 31 f., 35 f., 54, 83

Sterbetourismus $186 \mathrm{ff}$.

Steuergerechtigkeit 178

Stuttgart 23, 87, 328 f., 350 ff.

Südtirol 20 ff., $34 \mathrm{f}$.

Südtiroler Volkspartei 22, 27 ff.
Systemunverträglichkeit direkter Demokratie $49,56,60 \mathrm{f}$.

Tessin 19, 196

Texas 167

Thurgau 192, 195

Thüringen 51

Todesstrafe 163, 166, 169, 173 ff.

Tschechien 217

Umweltschutz $164 \mathrm{f}$.

Ungarn 45

Unterschriftensammlung $218 \mathrm{f}, 221 \mathrm{f}$.

Uri 192, $194 \mathrm{f}$.

USA 45 f., 65, 224, 243

US-Einzelstaaten 40, 41 f., 46 f., 50, $159 \mathrm{ff}$.

Verantwortungsdelegation $75 \mathrm{f}$.

Verbindlichkeit direktdemokratischer Entscheidungen $44 \mathrm{f}$., 49

Verfassungsgerichtsbarkeit 34 f., 41 f., 50 f., 59, 61, 84, 92, 94, 107, 109, 137 ff., 150, 153 f., 188 ff., 193, 259 f., 291 f., 321 ff., 385 f., 399 ff.

Verfassungskonvent 202 f., 208 f.

Verfassungsvergleich 45 f., 234 ff.

Vertragskündigung $370 \mathrm{ff}$.

Vertrauenspersonen $145 \mathrm{f}$.

Verwaltungsverfahrensgesetze $364 \mathrm{ff}$.

Völkerrecht 171 ff., 183

Volksabstimmung 20 ff., 240 f., 247 ff., $371 \mathrm{ff}$.

Volksanträge 90 f., 93

Volksbefragung 114, 135

Volksbegehren 135 f., 138 ff., 255 ff., $262 \mathrm{ff}$

Volksentscheid 135, 138 ff., 159 ff., 178 ff., 255 ff., 267 ff., 295 ff., 348 f.

Volksgesetzgebung 37 ff., 70, 74, 91 ff. 
Volksinitiative 70, 74, 90 f., 113, 160, 168, 177 ff., 258

- Allgemeine $169 \mathrm{ff}$.

- Europäische Bürgerinitiative $201 \mathrm{ff}$.

- Konsultative Gesetzesinitiative 57

- positive $43 \mathrm{f}$.

- Südtirol 22 ff., 29 ff.

- Verfassungsinitiative 34, 46, 160, 170

- Vetoinitiative 44 f., $58 \mathrm{ff}$.

- Vorlageninitiative 94

Volksmehr 53, 83
Wahlbeteiligung 79

Wallis 19

Washington $160 \mathrm{ff} ., 167 \mathrm{f}$.

Weimarer Reichsverfassung 47 f., 386 ff.

Werbung zur Teilnahme am Volksentscheid 349

Westminster-Modell $59 \mathrm{f}$.

Zug 192

Zürich 186 f., 191 f., 195 ff., 198

Zustimmungsquorum 82, 92, 136, 140, $148 \mathrm{f}$.

Zweite Kammer 52 f. 